\title{
$k$-REGULAR ELEMENTS IN SEMISIMPLE ALGEBRAIC GROUPS
}

\author{
BY
}

PETER P. ANDRE ${ }^{1}$ )

\begin{abstract}
In this paper, Steinberg's concept of a regular element in a semisimple algebraic group defined over an algebraically closed field is generalized to the concept of a $k$-regular element in a semisimple algebraic group defined over an arbitrary field of characteristic zero. The existence of semisimple and unipotent $k$-regular elements in a semisimple algebraic group defined over a field of characteristic zero is proved. The structure of all $k$-regular unipotent elements is given. The number of minimal parabolic subgroups containing a $k$ regular element is given. The number of conjugacy classes of $R$-regular unipotent elements is given, where $R$ is the real field. The number of conjugacy classes of $Q_{p}$-regular unipotent elements is shown to be finite, where $Q_{p}$ is the field of $p$-adic numbers.
\end{abstract}

1. Introduction. In [11], R. Steinberg defined a regular element in a semisimple algebraic group defined over an algebraically closed field to be one whose centralizer had minimal dimension. He showed that there exists one conjugacy class of unipotent regular elements and that a unipotent element was regular if and only if it was contained in a unique Borel subgroup. He also showed that a unipotent element $u$ was regular if and only if

$$
u=x_{\alpha_{1}} \cdots x_{\alpha_{n}} x_{\beta_{1}} \cdots x_{\beta_{m}}
$$

where $x_{\alpha_{i}} \neq e$ for all simple roots $\alpha_{i}$.

In this paper, we extend Steinberg's results to groups defined over a field $k$ of characteristic zero which is not necessarily algebraically closed. We define a $k$-regular element to be an element in the split radical of a minimal parabolic subgroup whose centralizer has the same dimension as the centralizer of a maximal split torus. If $k$ is algebraically closed, then a $k$-regular element is regular in Steinberg's sense.

We will show the existence of both semisimple and unipotent $k$-regular elements. We shall also see that a unipotent element $u$ is $k$-regular if

Received by the editors August 31, 1973.

AMS (MOS) subject classifications (1970). Primary 20G15.

Key words and phrases. Algebraic group, Lie algebra, minimal parabolic subgroup, maximal $k$-split torus, $k$-regular element.

( $\left.{ }^{1}\right)$ This paper constitutes part of the author's doctoral dissertation at Yale University (1970). 
and only if $u=x_{\alpha_{1}} \cdots x_{\alpha_{n}} x_{\beta_{1}} \cdots x_{\beta_{m}}$ where $x_{\alpha_{i}} \neq e$ for all restricted simple roots $\alpha_{i}$. The number of minimal parabolic subgroups containing a $k$ regular element $x$ will be shown to be $\left|W_{k}(G)\right| /\left|W_{k}\left(C^{0}\left(x_{s}\right)\right)\right|$ where $W_{k}(G)$ is the restricted Weyl group of $G$. We will show that there is one $G_{R}$-conjugacy class of $R$-regular unipotent elements if $G$ is adjoint or if $G$ is not split and is not one of three possible exceptions. A method for calculating the number of $G_{R}$-conjugacy classes of $R$-regular unipotent elements in a split group is given. For the $p$-adics, the number of conjugacy classes of $Q_{p}$-regular unipotent elements is shown to be finite.

In [6], Kostant and Rallis examined an equivalent concept to $k$-regularity in the $\mathfrak{p}$-space of a Cartan decomposition $\mathfrak{g}=\mathfrak{p}+\mathfrak{p}$ of a complex semisimple Lie algebra.

In [8], L. P. Rothschild discusses the $G$-conjugacy classes of $R$-regular nilpotent elements in a real Lie algebra which are the Lie algebra analogues to $R$ regular unipotent elements.

2. Notation. We will be concerned with a semisimple algebraic group $G$ defined over a field $k$ of characteristic zero. The group of $k$-rational points of $G$ will be denoted $G_{k}$. Associated with such an algebraic group is a Lie algebra $g$ whose $k$-rational points will be denoted $\boldsymbol{g}_{k}$. In general, Gothic letters will be used to denote Lie algebras of algebraic subgroups.

Throughout the paper we will use $P$ to denote a minimal parabolic subgroup of $G$. $S$ will represent a maximal $k$-split torus contained in $P$ and $U$ the maximal unipotent subgroup contained in $P$. The action of $S$ on $U$ gives rise to a restricted root system $\Delta_{k}$ on $S$. We will call the set of roots $\alpha$ such that the root group $U_{(\alpha)}$ is contained in $P$, the positive roots and denote the set $\Delta_{k}^{+}$. The set $\Pi_{k}$ such that every root in $\Delta_{k}^{+}$can be written uniquely as a positive integral combination of roots in $\Pi_{k}$ is called the set of simple roots.

The paper makes strong use of Morosov's lemma, which can be found in [4] or [5]. The results on reductive algebraic groups used in this paper can be found in [2], [3], or [13].

3. Simply written elements.

Definition 1. A Lie triple $[X, H, Y]$ is a subset of three elements $\{X, H, Y\}$ of a Lie algebra such that $[H, X]=2 X,[H, Y]=-2 Y$ and $[X, Y]=H$.

Proposition 1. Let $X$ be a nilpotent element in $8_{k}$, the $k$-rational points of semisimple Lie algebra 8 . Then there exists a Lie triple, $[X, H, Y]$ such that $H \in 8_{k}$, the $k$-rational points of the Lie algebra of a maximal $k$ split torus of $G$. 
Proof. Since the characteristic of $g_{k}$ is zero, $X$ can be embedded in a Lie triple $\left[X, H^{\prime}, Y^{\prime}\right]$ in $g_{k}$. The Lie algebra $g_{k}^{\prime}$ spanned over $k$ by $\left\{X, H^{\prime}, Y^{\prime}\right\}$ is semisimple and thus forms the $k$-rational points of an algebraic Lie algebra $g^{\prime}$ by Corollary 7.9,p. 195 of [2]. Let $G^{\prime}$ be the group whose Lie algebra is $g^{\prime}$. The Lie algebra $\mathfrak{n}^{\prime}$ spanned by $X$ is the Lie algebra of a unipotent group $U^{\prime}$ in $G^{\prime}$. The normalizer of $n^{\prime}$ in $G^{\prime}$ is a Borel subgroup of $G^{\prime}$ and contains a maximal $k$-split torus $S^{\prime}$ of $G^{\prime}$. Let $S$ be a maximal $k$-split torus of $G$ containing $S^{\prime}$. Let $g$ be the Lie algebra of $S$ and $\boldsymbol{g}^{\prime}$ be the Lie algebra of $S^{\prime}$. Since ad $\varepsilon^{\prime}$ normalizes $n^{\prime}$, this defines a root $\alpha$ on $8^{\prime}$. Let $\mathfrak{n}^{\prime \prime}$ be the root space in $\mathfrak{g}^{\prime}$ for the root $-\alpha$. The elements of $\mathfrak{n}^{\prime \prime}$ are nilpotent elements of $g^{\prime}$. The spaces $\mathfrak{n}^{\prime}, \mathfrak{g}^{\prime}$ and $\mathfrak{n}^{\prime \prime}$ span $\boldsymbol{g}^{\prime}$. Let $H \in \boldsymbol{g}_{k}^{\prime}$ be such that $[H, X]=2 X$. Thus $[H, N]=-2 N$ for any $N \in \mathrm{n}_{k}^{\prime \prime}$.

$$
[[N, X], H]=-[[X, H], N]-[[H, N], X]=2[X, N]-2[X, N]=0 .
$$

Thus $[N, X] \in \bar{g}_{k}^{\prime}$. Pick $Y \in \mathfrak{n}_{k}^{\prime \prime}$ such that $[X, Y]=H$. Thus $[X, H, Y]$ is a Lie triple and $H \in g_{k}^{\prime} \subset g_{k}$ where $\delta$ is the Lie algebra of a maximal $k$-split torus.

For the remainder of this section, $P$ will be a fixed minimal parabolic subgroup and $S$ a fixed maximal $k$-split torus contained in $P$. $U$ will be the maximal unipotent subgroup of $P$. Then $U=U_{\left(\alpha_{1}\right)} U_{\left(\alpha_{2}\right)} \cdots U_{\left(\alpha_{r}\right)}$ where $U_{\left(\alpha_{i}\right)}$ is the $\alpha_{i}$-root group and $\alpha_{i}$ is a root with respect to $S$.

Definition 2. A nilpotent element $N \in \mathfrak{n}_{k}$, the $k$-rational points of the Lie algebra $\mathfrak{n}$ of the group $U$, is simply written if $N=\Sigma_{\alpha \in \Pi_{k}} X_{\alpha}$ where $X_{\alpha} \in \mathfrak{n}_{\alpha}, \alpha$-root space and $X_{\alpha} \neq 0$ for all $\alpha \in \Pi_{k}$.

Proposition 2. A simply written nilpotent element $N \in \mathfrak{n}_{k}$ is contained as the first member of a Lie triple $[N, H, Y]$ in $8_{k}$ such that $H \in 8_{k}$, the $k$-rational points of the Lie algebra of the maximal k-split torus $S$.

Proof. By Proposition $1, N$ can be embedded in a Lie triple $\left[N, H^{\prime}, Y^{\prime}\right]$ where $H^{\prime}$ is an element of the $k$-rational points of the Lie algebra $z^{\prime \prime}$ of a maximal $k$-split torus $S^{\prime \prime}$. Let $\mathfrak{n}^{\prime}$ be the $\bar{k}$-space spanned by $N$. Let $R\left(\mathfrak{n}^{\prime}\right)$ be the normalizer of $n^{\prime}$ in $G$. Let $x \in R\left(\mathfrak{n}^{\prime}\right)_{k}$. Then, by the Bruhat decomposition, $x=u_{1} n_{w} u_{2}$ where $u_{1}, u_{2} \in U_{k}$ and $n_{w} \in N(S)_{k}$. Since $x \in R\left(\mathfrak{n}^{\prime}\right)_{k}$, $u_{1} n_{w} u_{2} N u_{2}^{-1} n_{w}^{-1} u_{1}^{-1}=t N$ where $t \in k$. Let $u_{2} N u_{2}^{-1}=Y=\Sigma Y_{\alpha}+\Sigma Y_{\beta}$ with $Y_{\alpha} \neq 0$ for all $\alpha \in \Pi_{k}$. But

$$
n_{w} Y n_{w}^{-1}=\sum n_{w} Y_{\alpha} n_{w}^{-1}+\sum n_{w} Y_{\beta} n_{w}^{-1}=\sum Y_{w^{-1}(\alpha)}^{\prime}+\sum Y_{w^{-1}(\beta)}^{\prime}
$$

which is not in $\mathfrak{p}$, the Lie algebra of the minimal parabolic subgroup $P$, unless $w=e$ and $n_{w} \in P$. Thus $R\left(\mathfrak{n}^{\prime}\right)_{k} \subset P$ and $R\left(\mathfrak{n}^{\prime}\right) \subset P$. Since $H^{\prime}$ is in the Lie algebra of $R\left(\mathfrak{n}^{\prime}\right), H^{\prime} \in \mathfrak{p}$. 
$H^{\prime}$ is therefore contained in the Lie algebra of a maximal $k$-split torus contained in the minimal parabolic subgroup $\boldsymbol{P}$. The maximal $k$-split tori of $\boldsymbol{P}$ are conjugate by elements in $U_{k}$. Therefore, there is an element $u \in U_{k}$ such that $u H^{\prime} u^{-1} \in \delta_{k}$, the $k$-rational points of the Lie algebra 8 of the maximal $k$-split torus $S$. Now, $u N u^{-1}=\Sigma_{\alpha \in \Pi_{k}} X_{\alpha}+\Sigma X_{\beta}, \beta$ ranging over higher roots. Let $H=u H^{\prime} u^{-1}$. Then,

$$
\begin{aligned}
{\left[H, u N u^{-1}\right] } & =2 u N u^{-1}=\left[H, \sum X_{\alpha}+\sum X_{\beta}\right] \\
& =\sum \alpha(H) X_{\alpha}+\sum \beta(H) X_{\beta}=\sum 2 X_{\alpha}+\sum 2 X_{\beta} .
\end{aligned}
$$

But, then $\alpha(H)=2$ for all $\alpha \in \Pi_{k}$. Thus $\beta(H)>2$ for all higher roots. But $\beta(H)=2$ unless $X_{\beta}=0$ for all $\beta$, and $u N u^{-1}=N$. Therefore, the Lie triple $\left[N, H, u Y^{\prime} u^{-1}\right]$ is the Lie triple which we want.

COROLlaRY 1. Let $N \in \mathfrak{n}_{k}$ be simply written. In the Lie triple $[N, H, Y]$ such that $H \in \mathfrak{n}_{k}, H$ and $Y$ are unique.

Proof. If $N=\Sigma X_{\alpha}, \alpha \in \Pi_{k}$. Then $\alpha(H)=2$ for all $\alpha \in \Pi_{k}$. Since the simple roots form a basis for $\delta_{k}^{*}, H$ is uniquely determined. $Y$ is unique from p. 984 in [5].

\section{4. $k$-split elements.}

DEFINITION 3. The split radical $S R(P)$ of a minimal parabolic subgroup $P$ is the group generated by a maximal split torus $S$ and the unipotent radical $U$ of $P$.

Definition 4. An element $g \in G_{k}$ is a $k$-split element if it is contained in the split radical of a minimal parabolic subgroup.

LEMMA 1. Every k-rational element of $S R(P)$ is conjugate by an element in $U_{k}$ to an element of the form of su where $s \in S_{k}$ and $u \in U_{k}$ and $s$ commutes. with $u$.

Proof. Order the positive roots $\Delta_{k}^{+}$starting with roots of height one and then height two and so on. Let $x \in S R(P)_{k}$. Then $x=s_{1} u_{1}$ where $s_{1} \in S_{k}$ and $u_{1}=\Pi x_{\alpha_{i_{i}}} \in U_{k}$ where $x_{\alpha_{i}} \in U_{\alpha_{i_{k}}}$, the $k$-rational points of the $\alpha_{i}$-root group, and $\alpha_{i}<\alpha_{j}$ if $i<j$.

If $s_{1}$ commutes with $x_{\alpha_{1}}$, proceed to $x_{\alpha_{2}}$. If $s_{1}$ does not commute with $x_{\alpha_{1}}$, then $\alpha_{1}\left(s_{1}\right) \neq 1$. If $x_{\alpha_{1}}=\exp \left(X_{\alpha_{1}}\right)$, construct

$$
u_{\alpha_{1}}=\exp \left(\frac{\alpha_{1}\left(s_{1}\right)}{1-\alpha_{1}\left(s_{1}\right)} X_{\alpha_{1}}\right) \text {. }
$$




$$
\begin{aligned}
s_{1}^{-1} u_{\alpha_{1}} s_{1} u_{\alpha_{1}}^{-1} & =s_{1}^{-1} \exp \left(\frac{\alpha_{1}\left(s_{1}\right)}{1-\alpha_{1}\left(s_{1}\right)} X_{\alpha_{1}}\right) s_{1} \exp \left(\frac{\alpha_{1}\left(s_{1}\right)}{\alpha_{1}\left(s_{1}\right)-1} X_{\alpha_{1}}\right) \\
& =\exp \left(\frac{-1}{\alpha_{1}\left(s_{1}\right)-1} X_{\alpha_{1}}\right) \exp \left(\frac{\alpha_{1}\left(s_{1}\right)}{\alpha_{1}\left(s_{1}\right)-1} X_{\alpha_{1}}\right)=\exp \left(X_{\alpha_{1}}\right)=x_{\alpha_{1}} .
\end{aligned}
$$

Thus $u_{\alpha_{1}}^{-1} s_{1} x_{\alpha_{1}} u_{\alpha_{1}}=s_{1}$ and $u_{\alpha_{1}}^{-1} s_{1} u_{1} u_{\alpha_{1}}=s_{1} x_{\alpha_{2}}^{\prime} \cdots x_{\alpha_{n}}^{\prime}$.

Proceeding inductively, $s_{1} u_{1}$ is conjugate by an element in $U_{k}$ after $r$ steps to $s_{1} \Pi x_{\beta_{i}}^{\prime} \Pi x_{\gamma_{j}}^{\prime}$ where $x_{\beta_{i}}^{\prime}$ commutes with $s_{1}$ and $\gamma_{j}>\alpha_{i}$ for all $\alpha_{i}, 1 \leqslant i \leqslant r$.

PROPOSITION 3. Every semisimple $k$-split element is contained in a maximal $k$-split torus.

Proof. Let $x$ be a semisimple element of $S R(P)_{k}$. By Lemma $1, x$ is conjugate by $u_{1}$ in $U_{k}$ to an element of the form $s u$ where $s \in S_{k}$ and $u \in U_{k}$ and $s u=u s$. But by the uniqueness of the Jordan decomposition, $u=e$ and $u_{1} x u_{1}^{-1}=$ $s_{1}$ since $u_{1} x u_{1}^{-1}$ is semisimple. Thus $x$ is an element of the maximal $k$-split torus $u_{1}^{-1} S u_{1}$.

\section{5. $k$-regular elements.}

Definition 5. A $k$-split element $x$ is $k$-regular if $\operatorname{dim} C(x)=\operatorname{dim} C(S)$ where $C(x)$ is the centralizer of $x$ in $G$ and $C(S)$ is the centralizer of $S$ in $G$.

PROPOSITION 4. If $x$ is a $k$-regular semisimple element and $y$ is a $k$-split semisimple element then $\operatorname{dim} C(x) \geqslant \operatorname{dim} C(y)$.

Proof. By Proposition 3, every semisimple $k$-split element is contained in a maximal $k$-split torus. Thus $y$ is an element of a $k$-split torus $S_{1}$. But, $\operatorname{dim} C(y) \leqslant$ $\operatorname{dim} C\left(S_{1}\right)=\operatorname{dim} C(S)=\operatorname{dim} C(x)$.

PROPOSITION 5. There exists a $k$-regular semisimple element.

Proof. Since $C^{0}(x)$ is reductive, where $C^{0}(x)$ is the connected component of the identity and $x$ is an element of $S_{k}, C^{0}(x)$ is generated by $C(s)$ and the root groups $U_{(\alpha)}$ where $\alpha(x)=1$. Since $S$ is $k$-split, $S_{k} \cong \operatorname{Hom}\left(X^{*}(S), k^{*}\right)$. Since $k$ is infinite, there exists $s \in S_{k}$ such that $\alpha(s) \neq 1$ for all $\alpha \in X^{*}(S)$. Therefore, $\alpha(s) \neq 1$ for all $\alpha \in \Delta_{k}$. Therefore, $C^{0}(s)=C(S)$ and $s$ is $k$-regular.

Definition 6. A unipotent element $u \in U_{k}$ is simply written with respect to a maximal $k$-split torus $S$, if $u=\exp (N)$ and $N$ is a simply written nilpotent element.

PROPOSITION 6. There exists a $k$-regular unipotent element.

Proof. Assume that $G$ is not anisotropic. Let $x$ be a simply written unipotent element of $U_{k}$ with respect to the maximal $k$-split torus $S$. Then $x=$ 
$\exp (X)$ where $X=\Sigma X_{\alpha}, \alpha \in \Pi_{k}$ and $X_{\alpha} \neq 0$. By Proposition 2, $X$ is contained in a Lie triple $[X, H, Y]$ with $H \in \delta_{k}$, the $k$-rational points of the Lie algebra of the group $S$. Let $a$ be the $k$-Lie algebra spanned by $\{X, H, Y\} . g_{k}$ is a completely reducible $a$-module. Therefore, we can write $\boldsymbol{g}_{k}$ as the sum of irreducible a-modules $\boldsymbol{g}_{k}^{(i)}$. Each $\boldsymbol{g}_{k}^{(i)}$ is the direct sum of eigenspaces $\left(g_{-m_{i}+2 p}^{(i)}\right)_{k}$ of $H$ such that if $N \in\left(g_{-m_{i}+2 p}^{(i)}\right)_{k}$, then $[H, N]=\left(-m_{i}+2 p\right) N$.

$\operatorname{dim} C(X)=n$, the number of irreducible components. $C(H)$ has a onedimensional intersection with $g^{(i)}$ if $m_{i}$ is even. If $m_{i}$ is odd, $C(H) \cap$ $g^{(i)}=\{0\}$.

Now, $2 X=[H, X]=\left[H, \Sigma X_{\alpha}\right]=\Sigma \alpha(H) X_{\alpha}$. Thus $\alpha(H)=2$ for all $\alpha \in \Pi_{k}$ and $\beta(H)$ is even for all $\beta \in \Delta_{k}^{+}$. Thus $m_{i}$ cannot be odd for any $i$ Therefore, $\operatorname{dim} C(H)=\operatorname{dim} C(X)=n$.

But, $\beta(H) \neq 0$ for any $\beta \in \Delta_{k}^{+}$. Thus $\operatorname{dim} C(H)=\operatorname{dim} C(\xi)=\operatorname{dim} C(S)$. Since $C(X)$ is the Lie algebra of $C(x), \operatorname{dim} C(x)=\operatorname{dim} C(X)=\operatorname{dim} C(H)=$ $\operatorname{dim} C(S)$. Therefore, $x$ is $k$-regular.

If $G$ is anistropic, then the identity is a $k$-regular unipotent element.

Proposition 7. Let $x$ be a $k$-regular unipotent element and $y$ a unipotent element in $G_{k}$. Then $\operatorname{dim} C(k) \leqslant \operatorname{dim} C(y)$.

Proof. Let $y=\exp Y$. By Proposition $1, Y$ is contained in a Lie triple $[Y, H, Z]$ such that $H \in 8_{k}^{\prime}$, the $k$-rational points of the Lie algebra of a maximal $k$-split torus $S^{\prime}$. Let a be the $k$-Lie algebra generated by $\{Y, H, Z\}$. As in Proposition $6, g_{k}$ is the direct sum of irreducible $a$-modules $\boldsymbol{g}_{k}^{(i)}$. But $\operatorname{dim} C(H) \leqslant \operatorname{dim} C(Y)$ and $\operatorname{dim} C(x)=\operatorname{dim} C(S)=\operatorname{dim} C\left(S^{\prime}\right) \leqslant \operatorname{dim} C(H)$. Thus $\operatorname{dim} C(x) \leqslant \operatorname{dim} C(Y)=\operatorname{dim} C(y)$.

Proposition 8. A k-split element $x$ is $k$-regular if and only if $x_{u}$ is $k$ regular in $C^{0}\left(x_{s}\right)$, the Zariski component of the identity in $C\left(x_{s}\right)$ where $x=$ $x_{s} x_{u}$ is the Jordan decomposition of $x$.

Proof. We know that $C(x) \subseteq C\left(x_{s}\right)$, since anything which commutes with $x$ commutes with both its semisimple and unipotent parts. Thus, the Zariskiconnected component of $C(x)$ containing the identity is equal to $C_{H}^{0}\left(x_{u}\right)$, the Zariski-connected component of the centralizer of $x_{u}$ in $C^{0}\left(x_{s}\right)$, which we denote by $H$. Let $x$ be $k$-regular. Then it is $k$-split and thus $x_{s}$ and $x_{u}$ are $k$-split. Therefore, $x_{s}$ is contained in a maximal $k$-split torus $S$ and $S \subseteq C^{0}\left(x_{s}\right)$ $=H$. Since $x_{s} \in S$, then $C(S) \subseteq C^{0}\left(x_{s}\right)=H$ and $\operatorname{dim} C_{G}(S)=\operatorname{dim} C_{H}(S)$. Since $\operatorname{dim} C_{G}(x)=\operatorname{dim} C_{H}(x), x$ is $k$-regular in $H$. But, $\operatorname{dim} C_{H}(x)=$ $\operatorname{dim} C_{H}\left(x_{u}\right)$ and $x_{u}$ is $k$-regular in $H=C^{0}\left(x_{s}\right)$.

Suppose $x_{u}$ is $k$-regular in $C^{0}\left(x_{s}\right)$. Then $\operatorname{dim} C_{G}(x)=\operatorname{dim} C_{H}(x)=$ 
$\operatorname{dim} C_{H}\left(x_{u}\right)=\operatorname{dim} C_{H}(S)=\operatorname{dim} C_{G}(S)$ and $x$ is $k$-regular in $G$.

COROllary 2. Every semisimple k-split element is the semisimple part of $a$ k-regular element.

Proof. Let $x_{s}$ be a semisimple $k$-split element. By Proposition 6, there exists a $k$-regular unipotent element $x_{u}$ in $C^{0}\left(x_{s}\right)$. The element $x_{s} x_{u}$ is $k$ regular in $G$ and $x=x_{s} x_{u}$ is the Jordan decomposition.

\section{6. $k$-regular unipotent elements.}

Proposition 10. A unipotent element $u \in U_{k}$ is k-regular if $u=$ $x_{\alpha_{1}} x_{\alpha_{2}} \cdots x_{\alpha_{n}} x_{\beta_{1}} \cdots x_{\beta_{m}}$ where $x_{\alpha_{i}} \in U_{\alpha_{i}}$ the $\alpha_{i}$ root group, $x_{\beta_{i}} \in U_{\beta_{i}}$ the $\beta_{i}$ root group, $\alpha_{i} \in \Pi_{k}$ and $x_{\alpha_{i}} \neq e$ for all $\alpha_{i} \in \Pi_{k}$ and $x_{\alpha_{i}} \notin U_{2 \alpha_{i}}$ if $2 \alpha_{i}$ is a root, $\beta_{i}$ range over positive roots of higher order.

Proof. It suffices to show that $u$ is $G_{k}$-conjugate to a simply written unipotent element $x$.

Let $u=\exp N$ where $N=\Sigma X_{\alpha}+\Sigma X_{\beta}$ where $\alpha \in \Pi_{k}$ and ranges over positive roots of height greater than one. We will show that $N$ is $G_{k}$-conjugate to $X=\Sigma X_{\alpha}$. Note that $X_{\alpha} \neq 0$ for all $\alpha \in \Pi_{k}$. The conjugation will be carried out sequentially. $X$ will be conjugated by an element in $U_{k}$ so that it will have the same components of $N$ in the root spaces of height one and two and then that element will be conjugated so that it agrees with $N$ in all root spaces of height one, two and three and so on.

Let $\mathfrak{n}_{k}$ be the $k$-rational points of the Lie algebra $\mathfrak{n}$ of $U$. Let $\mathfrak{n}_{k}^{+}$be the $k$-rational points of the Lie algebra $\mathfrak{n}^{+}$spanned by the root spaces $\mathfrak{n}_{\beta_{i}}$ where $\beta_{i}$ has height greater than one. We shall first prove that $\left[X, n_{k}\right]=\mathfrak{n}_{k}^{+}$.

Since $X$ is simply written it can embedded in a Lie triple $[X, H, Y]$ such that $H \in \mathbb{B}_{k}$, the Lie algebra of $S$. Let $a$ be the $k$-Lie algebra spanned by $[X, H, Y]$. Then $g_{k}$ is the direct sum of irreducible $a$-modules $\mathfrak{g}_{k}^{(i)}$. Each $g_{k}^{(i)}$ is the direct sum of eigenspaces $\left(g_{-m_{i}+2 p}^{(i)}\right)_{k}$ where $[H, Z]=\left(-m_{i}+2 p\right) Z$ if $Z \in\left(g_{-m_{i}+2 p}^{(i)}\right)_{k}$. Let $g_{R_{k}}=\Sigma_{i=1}^{n}\left(g_{R}^{(i)}\right)_{k}$. Note that, for $R>0,\left[X, g_{R_{k}}\right]=$ $\left(g_{R+2}\right)_{k}$. Let $g_{k}^{+}=\Sigma_{R>0}\left(g_{R}\right)_{k}$ and $g_{k}^{++}=\Sigma_{R>2}\left(g_{R}\right)_{k}$. Therefore, $\left[X, g_{k}^{+}\right]=$ $\mathfrak{g}_{k}^{++}$. Since $X$ is simply written, $X=\Sigma X_{\alpha}, \alpha \in \Pi_{k}$. Then $[H, X]=\left[H, \Sigma X_{\alpha}\right]=$ $\Sigma \alpha(H) X_{\alpha}=2 \Sigma X_{\alpha}$. Therefore, $\alpha(H)=2$ for all simple roots $\alpha$, and $\beta(H)$ is positive and even for all positive roots $\beta \in \Delta_{k}^{+}$. Thus $\mathfrak{n}_{k} \subset \boldsymbol{g}_{k}^{+}$and $\mathfrak{n}_{k}^{+} \subset \boldsymbol{g}_{k}^{++}$ but $\operatorname{dim} \mathfrak{n}_{k} \geqslant \operatorname{dim} \mathfrak{g}_{k}^{+}$and $\operatorname{dim} \mathfrak{n}_{k}^{+} \geqslant \operatorname{dim} \mathfrak{g}_{k}^{++}$. Therefore, $\mathfrak{n}_{k}=\boldsymbol{g}_{k}^{+}$and $\mathfrak{n}_{k}^{+}=\mathfrak{g}_{k}^{++}$. Thus $\left[X, \mathfrak{n}_{k}\right]=\mathfrak{n}_{k}^{+}$.

Let $N_{i}$ be the sum of all $X_{\beta_{j}}$ in the expansion of $U$ into components such that the height of $\beta_{j}$ is $i$ By above, there exists $Y_{2} \in \mathfrak{n}_{k}$ such that $\left[X, Y_{2}\right]=N_{2}$ and 


$$
\begin{aligned}
X_{2} & =\exp \left(Y_{2}\right) X \exp \left(-Y_{2}\right)=X+\left[X, Y_{2}\right]+\text { higher terms } \\
& =X+N_{2}+X_{3}^{(2)}+X_{4}^{(2)}+\cdots
\end{aligned}
$$

where $X_{i}^{(2)}$ is the component of $\exp \left(Y_{2}\right) X \exp \left(-Y_{2}\right)$ in the root spaces of roots of height $i$.

There exists a $Y_{3} \in \mathfrak{n}_{k}$ such that $\left[X, Y_{3}\right]=N_{3}-X_{3}^{(2)}$. Then

$$
\begin{aligned}
\exp \left(Y_{3}\right) X_{2} \exp \left(-Y_{3}\right) & =X_{2}+\left[X_{2}, Y_{3}\right]+\text { higher terms } \\
& =X+N_{2}+X_{3}^{(2)}+N_{3}-X_{3}^{(2)}+\text { higher terms } \\
& =X+N_{2}+N_{3}+\text { higher terms. }
\end{aligned}
$$

Proceeding sequentially we can conjugate $X$ by a suitable element in $U_{k}$ and obtain $N$. Thus $u=\exp (N)$ is $G_{k}$-conjugate to $x=\exp (X)$ and $u$ is $k$-regular.

Proposition 11. Let $u$ be a $k$-regular element in $U_{k}$. The $G_{k}$-conjugacy class of $u$ is dense in $U$.

PRoof. Let $\phi: P \rightarrow U$ be defined by $\phi(p)=p u p^{-1}$. Since $\phi$ is a regular, $\phi(p)$ contains an open subset of $\overline{\phi(P)}$. However,

$$
\begin{aligned}
\operatorname{dim} C(S) & =\operatorname{dim} C_{G}(u) \geqslant \operatorname{dim} C_{P}(u) \\
& =\operatorname{dim}\left(\phi^{-1}(u)\right) \geqslant \operatorname{dim} P-\operatorname{dim} \overline{\phi(P)} .
\end{aligned}
$$

But $\operatorname{dim} P=\operatorname{dim} C(S)+\operatorname{dim} U$. Thus $\operatorname{dim} \overline{\phi(P)}=\operatorname{dim} U$ and $\overline{\phi(P)}=U$. Since $P_{k}$ is dense in $P, \phi\left(P_{k}\right)$ is dense in $\overline{\phi(P)}$. Thus $\phi\left(P_{k}\right)$ is dense in $U$.

Proposition 12. Let $u \in U_{k}$ such that $u=x_{\alpha_{1}} x_{\alpha_{2}} \cdots x_{\alpha_{n}} x_{\beta_{1}} \cdots x_{\beta_{m}}$, $\alpha_{i} \in \Pi_{k}$ and $x_{\alpha_{j}}=e$ for some $\alpha_{j} \in \Pi_{k}$ and $\beta_{i}$ range over higher roots. Then $u$ is not $k$-regular.

Proof. Suppose $u$ is $k$-regular. The $P_{k}$-conjugacy class of $u$ is then dense in $U$ and contains an element $x$ such that $x=x_{\alpha_{1}} x_{\alpha_{1}} \cdots x_{\alpha_{n}} x_{\beta_{1}}$ $\cdots x_{\beta_{m}}$ and $x_{\alpha_{i}} \neq e$ for all $\alpha_{i} \in \Pi_{k}$. However, no $P_{k}$ conjugate of $u$ can have a nonidentity $\alpha_{j}$ component. Thus $u$ is not $k$-regular.

COROllary 3. A unipotent element $u \in U_{k}$ is $k$-regular if and only if $u=x_{\alpha_{1}} x_{\alpha_{2}} \cdots x_{\alpha_{n}} x_{\beta_{1}} \cdots x_{\beta_{m}}$ where $x_{\alpha_{i}} \neq e$ for all $\alpha_{i} \in \Pi_{k}$ and $\beta_{i}$ range over higher roots.

7. $k$-regular elements and minimal parabolic subgroups.

LEMMA 3. Let $x$ be a k-regular element which is a member of two minimal parabolic subgroups $P_{1}$ and $P_{2}$. Let $x$ be an element of the split radical 
$S R\left(P_{1}\right)$ of $P_{1}$ and $x_{s}$ be an element of $S$, a maximal $k$-split torus contained in $P_{1}$. Then there exists an element $n$ of $N(S)_{k}$, the $k$-rational points of the normalizer of $S$, such that $n P_{2} n^{-1}=P_{1}$.

Proof. Since $P_{1}$ and $P_{2}$ are minimal parabolic subgroups, there exists $g \in G_{k}$ such that $g P_{2} g^{-1}=P_{1}$. By the Bruhat decomposition, $g=u_{1} n_{w} u_{2}$ where $u_{1}, u_{2} \in R U\left(P_{1}\right)$ and $n_{w} \in N(S)_{k}$. Since $u_{1}$ is an element of $P_{1}$, $n_{w} u_{2} P_{2} u_{2}^{-1} n_{w}^{-1}=P_{1}$.

Let $u=x_{\alpha_{1}} x_{\alpha_{2}} \cdots x_{\alpha_{n}} x_{\beta_{1}} \cdots x_{\beta_{m}}$ where $w^{-1}\left(\alpha_{i}\right)$ is positive and $w^{-1}\left(\beta_{i}\right)$ is negative. Then

$$
\begin{aligned}
& n_{w} u_{2}=n_{w} x_{\alpha_{1}} x_{\alpha_{2}} \cdots x_{\alpha_{n}} x_{\beta_{1}} \cdots x_{\beta_{m}} \\
& =x_{w^{-1}\left(\alpha_{1}\right)} x_{w^{-1}\left(\alpha_{2}\right)} \cdots x_{w^{-1}\left(\alpha_{n}\right)^{n}} n_{w_{\beta_{1}}} \cdots x_{\beta_{m}}=u_{3} n_{w} u_{4} .
\end{aligned}
$$

But then $n_{w} u_{4} P_{2} u_{4}^{-1} n_{w}^{-1}=P_{1}$.

Let $\Delta_{k}^{\prime}=\left\{\alpha \in \Delta_{k} \mid \alpha\left(x_{s}\right)=1\right\} . \Delta_{k}^{\prime}$ is a root system for $C^{0}\left(x_{s}\right)$ and since $x$ is $k$-regular, $x_{u}$ is a $k$-regular unipotent element in $C^{0}\left(x_{s}\right)$. If $\Pi_{k}^{\prime}$ is a set of simple roots for $\Delta_{k}^{\prime}$ such that $\Pi_{k}^{\prime} \subset \Delta_{k}^{+}$, then $x_{u}=x_{\gamma_{1}} x_{\gamma_{2}} \cdots$ $x_{\gamma_{r}} x_{\delta_{1}} \cdots x_{\delta_{p}}$ where $x_{\gamma_{i}} \neq e$ for all $\gamma_{i} \in \Pi_{k}^{\prime}$ and $\delta_{i}$ ranges over the higher roots of $\Delta_{k}^{\prime+}=\Delta_{k}^{+} \cap \Delta_{k}^{\prime}$.

Suppose $\beta_{i}\left(x_{s}\right) \neq 1$ for some root $\beta_{i}$ where $x_{\beta_{i}} \neq e$ in the decomposition of $u_{4}$. Then pick the root $\beta_{j}$ of lowest height such that $\beta_{j}\left(x_{s}\right) \neq 1$. By the above, we have

$$
\begin{aligned}
& n_{w} x_{\beta_{1}}\left(t_{1}\right) x_{\beta_{2}}\left(t_{2}\right) \cdots x_{\beta_{n}}\left(t_{n}\right) x_{s} x_{u} x_{\beta_{n}}\left(-t_{n}\right) \cdots x_{\beta_{1}}\left(-t_{1}\right) n_{w} \\
& =n_{w} x_{s} x_{\beta_{1}}\left(t_{1} / \beta_{1}\left(x_{s}\right)\right) \cdots x_{\beta_{n}}\left(t_{n} / \beta_{n}\left(x_{s}\right)\right) x_{u} x_{\beta_{n}}\left(-t_{n}\right) \cdots x_{\beta_{1}}\left(-t_{1}\right) .
\end{aligned}
$$

Since $\beta_{j}$ is the root of lowest height such that $\beta_{j}\left(x_{s}\right) \neq 1$, it is not a positive integral combination of the other $\beta_{i}$ 's, $\gamma_{i}$ 's and $\delta_{i}$ 's. Therefore, the $\beta_{j}$ component of $u_{4} x_{5} x_{u} u_{4}^{-1}$ is not the identity, since

$$
x_{\beta_{j}}\left(t_{j} / \beta_{j}(x)\right) x_{\beta_{j}}\left(-t_{j}\right)=x_{\beta_{j}}\left(t_{j}\left(\frac{1-\beta_{j}\left(x_{s}\right)}{\beta_{j}\left(x_{s}\right)}\right)\right) \neq e .
$$
then

But if $u_{4} x_{5} x_{u} u_{4}^{-1}$ is written $x_{5} x_{\rho_{1}} \cdots x_{\rho_{q}}$ where $\rho_{i} \in \Delta_{k}^{+}, \rho_{1}=\beta_{j}$,

$$
n_{w} u_{4} x_{s} x_{u} u_{4}^{-1} n_{w}^{-1}=x_{s}^{\prime} x_{w}^{\prime}{ }^{-1}\left(\rho_{1}\right) \cdots x_{w^{-1}\left(\rho_{q}\right)}^{\prime} \text { where } n_{w} x_{s} n_{w}^{-1}=x_{s}^{\prime} .
$$

But $n_{w} u_{4} x_{s} x_{u} u_{4}^{-1} n_{w}^{-1}$ is an element of $P_{1}$, while $w^{-1}\left(\rho_{1}\right)$ is negative and 
$x_{w^{-1}\left(\rho_{1}\right)}^{\prime}$ is not in $P_{1}$. Therefore, by contradiction, $\beta_{i}\left(x_{s}\right)=1$ for all roots $\beta_{i}$ such that $x_{\beta_{i}} \neq e$ in the expansion of $u_{4}$.

Assume that there is a $\beta_{j}$ such that $x_{\beta_{j}} \neq e$ in the expansion of $u_{4}$. Then $\beta_{j}$ is a positive integral combination of the roots in $\Pi_{k}^{\prime}$, since $\beta_{j}\left(x_{s}\right)=1$. But $w^{-1}\left(\beta_{j}\right)$ is negative, thus $w^{-1}\left(\gamma_{i}\right)$ is negative for some $\gamma_{i} \in \Pi_{k}^{\prime}$. Since $x_{u}$ is $k$-regular in $C^{0}\left(x_{s}\right), x_{\gamma_{i}} \neq e$. Since $\gamma_{i}$ is simple in $\Delta_{k}^{\prime+}$, the $\gamma_{i}$ component of $u_{4} x_{u} u_{4}^{-1}$ is not the identity. Thus

$$
\begin{aligned}
n_{w} u_{4} x_{s} x_{u} u_{4}^{-1} n_{w}^{-1} & =x_{s}^{\prime} n_{w} x_{\gamma_{1}}^{\prime} \cdots x_{\gamma_{r}}^{\prime} x_{\delta_{1}}^{\prime} \cdots x_{\delta_{p}}^{\prime} n_{w} \\
& =x_{s}^{\prime} x_{w^{\prime}}^{\prime \prime}{ }_{\left(\gamma_{1}\right)} \cdots x_{w^{-1}\left(\gamma_{r}\right)}^{\prime \prime}{ }_{w^{-1}\left(\delta_{1}\right)}^{\prime \prime} \cdots x_{w^{-1}\left(\delta_{p}\right)}^{\prime \prime},
\end{aligned}
$$

and $x_{w^{-1}}^{\prime \prime}\left(\gamma_{j}\right)$ is not an element of $P_{1}$. This leads to a contradiction since $n_{w} u_{4} x u_{4}^{-1} n_{w}^{-1}$ is an element of $P_{1}$. Therefore $x_{\beta_{j}}=e$ for all $\beta_{j}$ and $n_{w} P_{2} n_{w}^{-1}=P_{1}$.

Proposition 13. A k-regular unipotent element is contained in only one minimal parabolic subgroup.

Proof. Let $u$ be a unipotent $k$-regular element such that $u$ is an element of two minimal parabolic subgroups $P_{1}$ and $P_{2}$. Then $u=e u$ is the Jordan decomposition of $u$ and $e$ is an element of every maximal $k$-split torus of $P_{1}$ and $u$ is an element of $S R\left(P_{1}\right)$. By Lemma 3, there exists $n_{w} \in N\left(S_{k}\right.$ such that $n_{w} P_{2} n_{w}^{-1}=P_{1}$. Let $u=x_{\alpha_{1}} x_{\alpha_{2}} \cdots x_{\alpha_{n}} x_{\beta_{1}} \cdots x_{\beta_{m}}$ where $x_{\alpha_{i}} \neq e$ for all $\alpha_{i} \in \Pi_{k}$ and $\beta_{i}$ range over higher roots. If $w \neq e$, then $w^{-1}\left(\alpha_{i}\right)$ is negative for some $\alpha_{i} \in \Pi_{k}$ and $x_{w^{-1}\left(\alpha_{i}\right)}^{\prime}$ is not an element of $P_{1}$. But

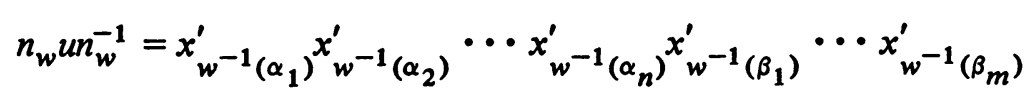

is an element of $P_{1}$. This is a contradiction. Hence, $w=e$ and $n_{w}$ is an element of $P_{1}$. Therefore $P_{1}=P_{2}$.

COROLLARY 5. The centralizer of a $k$-regular unipotent element is contained in $P$, the unique minimal parabolic subgroup containing $u$.

PROof. Let $g$ be an element of $G_{k}$ such that $g u g^{-1}=u$. Then $g g^{-1}$ is a minimal parabolic subgroup containing $u$. Thus $g P g^{-1}=P$ and $g$ is an element of $P$ since $P$ is its own normalizer. Since $C(u)_{k}$ is dense in $C(u)$, $C(u)$ is contained in $P$.

Proposition 14. A k-regular semisimple element is contained in $\left|W_{k}\right|$ minimal parabolic subgroups where $W_{k}$ is the restricted Weyl group. 
Proof. Let $s$ be a $k$-regular semisimple element contained in a maximal $k$-split torus $S \subseteq P_{1}$ and let $s$ be an element of $P_{2}$. Then by Lemma 3, there exists $n_{w} \in N(S)_{k}$ such that $n_{w} P_{2} n_{w}^{-1}=P_{1}$. Since the elements in $N(S)_{k}$ normalize $S, s$ is an element of $n P_{1} n^{-1}$ for all $n \in N(S)_{k}$. Since $N(S)_{k} \cap$ $P_{1}=C(S)_{k}$ and $P_{1}$ is its own normalizer, the number of minimal parabolic subgroups of the form $n_{w} P_{1} n_{w}^{-1}$ is $\left|N(S)_{k} / C(S)_{k}\right|=\left|W_{k}\right|$.

Lemma 4. Let $s$ be a semisimple $k$-split element. A minimal parabolic subgroup $P_{1}$ of $C^{0}(s)$ is equal to the intersection of $C^{0}(s)$ and a minimal parabolic subgroup $P_{2}$ of $G$ such that $s$ is an element of $\operatorname{SR}\left(P_{2}\right)$.

Proof. Let $g$ be an element of $C^{0}(s)$ and let $s$ be an element of the maximal $k$-split torus $S$ where $S$ is contained in the minimal parabolic subgroup $P$. Now by the Bruhat decomposition $g$ can be written uniquely as $u_{1} n_{w} u_{2}$ where $u_{1}$ and $u_{2}$ are elements of $U_{k} \subseteq P, n_{w}$ is an element of $N(S)_{k}$ and $u_{2}=x_{\alpha_{1}} x_{\alpha_{2}} \cdots x_{\alpha_{n}}$ with $\alpha_{i} \in \Delta_{k}^{+}$and $w^{-1}\left(\alpha_{i}\right)$ is negative. sgs ${ }^{-1}=$ $s u_{1} n_{w} u_{2} s^{-1}=s u_{1} s^{-1} s n_{w} s^{-1} s u_{2} s^{-1}=g$. But $s u_{1} s^{-1}$ is an element of $U_{k}$, $s n_{w} s^{-1}$ is an element of $N(S)_{k}$ and $s u_{2} s^{-1}=x_{\alpha_{1}}^{\prime} x_{\alpha_{2}}^{\prime} \cdots x_{\alpha_{n}}^{\prime}$ where $w^{-1}\left(\alpha_{i}\right)$ is negative. Hence by the uniqueness of the Bruhat decomposition, $s u_{1} s^{-1}=u_{1}$, $s n_{w} s^{-1}=n_{w}, s u_{2} s^{-1}=u_{2}$. Therefore, $C^{0}(s)$ is generated by $C(S)$ and the root groups $U_{(\alpha)}$ such that $\alpha(s)=1$.

$P \cap C^{0}(s)=P_{1}$ is a minimal parabolic subgroup of $C^{0}(s)$, since $P \cap C^{0}(s)$ is generated by $C(S)$ and the root groups $U_{(\alpha)}$ such that $\alpha(s)=1$ and $\alpha \in \Delta_{k}^{+}$. Given a root $\alpha \in \Delta_{k}^{\prime}=\left\{\alpha \in \Delta_{k} \mid \alpha(s)=1\right\}$ then either $U_{(\alpha)}$ is contained in $P_{1}$ or $U_{(-\alpha)}$ is contained in $P_{1}$.

Let $P_{1}^{\prime}$ be another minimal parabolic subgroup of $C^{0}(s)$. Then $P_{1}^{\prime}=$ $g P_{1} g^{-1}=g\left(P \cap C^{0}(s)\right) g^{-1}=g P g^{-1} \cap C^{0}(s)$ where $g$ is an element of $C^{0}(s)_{k}$. But $\mathrm{gPg}^{-1}$ is a minimal parabolic subgroup of $G$ and $\mathrm{gSg}^{-1}$ is a maximal $k$-split torus containing $s$.

Proposition 15. Let $x=x_{s} x_{u}$ be a $k$-regular element. Then $x$ is contained in $\left|W_{k}(G)\right| /\left|W_{k}\left(C^{0}\left(x_{s}\right)\right)\right|$ minimal parabolic subgroups of $G$.

Proof. Let $x$ be contained in $S R\left(P_{1}\right)$, let $x_{s}$ be contained in the maximal $k$-split torus $S$ in $P_{1}$ and let $x$ be contained in another minimal parabolic subgroup $P_{2}$. By Lemma 3, there exists $n_{w} \in N(S)_{k}$ such that $n_{w} P_{2} n_{w}^{-1}=P_{1}$. Thus any minimal parabolic subgroup containing $x$ also contains $S$. Hence, if $P_{2}$ is a minimal parabolic subgroup containing $x$ then $P_{2} \cap C^{0}\left(x_{s}\right)$ is a minimal parabolic subgroup of $C^{0}\left(x_{s}\right)$. By Proposition $8, x$ is $k$-regular in $G$ if and only if $x_{u}$ is $k$-regular in $C^{0}\left(x_{s}\right)$. Therefore, by Proposition 13, $x$ is contained in only one minimal parabolic subgroup of $C^{0}\left(x_{s}\right)$. There are $\left|W_{k}(G)\right|$ 
minimal parabolic subgroups of $G$ containing $S$ and $\left|W_{k}\left(C^{0}\left(x_{s}\right)\right)\right|$ minimal parabolic subgroups of $C^{0}\left(x_{s}\right)$ containing $S$. Since $C(S)$ is contained in $C^{0}\left(x_{s}\right)$ we can think of $W_{k}\left(C^{0}\left(x_{s}\right)\right)$ as a subgroup of $W_{k}(G)$. Given a coset of $W_{k}(G) / W_{k}\left(C^{0}\left(x_{s}\right)\right)$, there is a representative $n_{w}$ in $N(S)_{k}$ such that $n_{w} P_{1} n_{w}^{-1}$ contains $x$. Two such representatives come from the same coset in $N(S)_{k} / C(S)_{k}$. Therefore, the number of minimal parabolic subgroups containing $x$ is $\left|W_{k}(G)\right| /\left|W_{k}\left(C^{0}\left(x_{s}\right)\right)\right|$.

8. The conjugacy classes of $R$-regular unipotent elements. In the following section, the field $k$ will be restricted to the field of real numbers $R$.

Proposition 16. The $P_{R}$-conjugacy class of an $R$-regular unipotent element is open in $U_{R}$, in the real topology, where $U$ is the unipotent radical of the minimal parabolic subgroup $P$.

Proof. Let $u \in U_{R}$ be an $R$-regular unipotent element. Let $u=\exp (N)$. Then we see that $\operatorname{dim} C(u)=\operatorname{dim} C(N)=\operatorname{dim} C\left(S_{R}\right)=\operatorname{dim} C\left(8_{R}\right)$ where 8 is the Lie algebra of the maximal $R$-split torus $S$. Let $\mathfrak{p}$ be the Lie algebra of $P$ and let $\mathfrak{n}$ be the Lie algebra of $U$. Since $\mathfrak{n}_{R}$ is normal in $\mathfrak{p}_{R}$, ad $(N)\left(\mathfrak{p}_{R}\right) \subseteq$ $\mathfrak{n}_{R}$. But $\mathfrak{p}_{R}=C\left(\mathfrak{g}_{R}\right)+\mathfrak{n}_{R}$. Thus $\operatorname{ad}(N)\left(\mathfrak{p}_{R}\right)=\mathfrak{n}_{R}$. Therefore, $P_{R}$ operating by conjugacy on $u$ is open in $U_{R}$.

COROllaRY 6. The $P_{R}$-conjugacy class of a unipotent $R$-regular element $u \in U_{R}$ is a union of connected components of the set of $R$-regular elements in $U_{R}$.

DEFINITION 7. Let $L_{i}=\left\{u \in U_{R} \mid u=x_{\alpha_{1}} x_{\alpha_{2}} \cdots x_{\alpha_{n}} x_{\beta_{1}} \cdots x_{\beta_{m}}\right\}$ where $\alpha_{j} \in \Pi_{R}, \beta_{j} \in \Delta_{R}^{+}, \beta_{j} \notin \Pi_{R}$ and $x_{\alpha_{i}}=e$.

Note 1. The union of the $L_{i}$ is the full set of the non-R-regular elements in $U$. Thus the set of $R$-regular elements $R_{u}$ is equal to $U_{R}-\bigcup_{i=1}^{n} L_{i}$. Each $L_{i}$ partitions $U_{R}-L_{i}$ into two components if and only if $\operatorname{dim} U_{\left(\alpha_{i}\right)}=1$. We shall call one such component $M_{i}$ and the other $N_{i}$. The set of $\alpha_{i} \in \Pi_{R}$ such that $\operatorname{dim} U_{\left(\alpha_{i}\right)}=1$ will be denoted $M$.

DEFinition 8. Let $\operatorname{dim} U_{\left(\alpha_{i}\right)}=1$ and $A$ be a connected component of $R_{u} \cdot \rho_{i}(A)=1$ if $A$ is contained in $M_{i}$ and $\rho_{i}(A)=-1$ if $A$ is contained in $N_{i}$.

Proposition 17. Two R-regular unipotent elements of $U_{R}$ are $G_{R}$-conjugate if and only if they are $P_{R}$-conjugate.

PROof. Let $u_{1}$ and $u_{2}$ be two $R$-regular unipotent elements in $U_{R}$, and let $g u_{1} g^{-1}=u_{2}$ for some $g \in G_{R}$. Both $u_{1}$ and $u_{2}$ are contained in a unique minimal parabolic subgroup $P$. But $\mathrm{gPg}^{-1}$ is a minimal parabolic 
subgroup containing $u_{2}$. Thus $g \mathrm{Pg}^{-1}=P$. But since $P$ is its own normalizer $g \in P_{R}$.

Proposition 18. An R-regular unipotent element in a connected com-

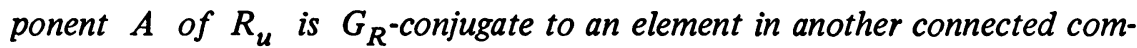
ponent $B$ of $R_{u}$ if and only if there exists an element $s \in S_{R}$ such that $\alpha_{i}(s) \in R^{+}$, the positive real numbers, if $\rho_{i}(A)=\rho_{i}(B)$ and $\alpha_{i}(s) \in R^{-}$, the real numbers, if $\rho_{i}(A) \neq \rho_{i}(B)$ for all $\alpha_{i} \in M$.

Proof. By Proposition 17, it is sufficient to restrict our discussion to $P_{R}$-conjugacy. Since conjugacy by elements in $U_{R}$ does not change the components of an element in the simple root spaces, it is sufficient to restrict our attention to $C(S)_{R}$-conjugacy.

Let $C(S)_{R}=D(C(S))_{R} S_{R}$ where $\mathcal{D}(C(S))$ is the derived group of $C(S)$ and $D(C(S))$ is an anisotropic semisimple algebraic group. Let $T^{\prime}$ be a maximal torus defined over $R$ in $D(C(S))$ and $T=T^{\prime} S$ is a maximal torus defined over $R$ in $G$. The restricted roots of $S$ are the restrictions of roots on $T$ to $S$. Since $\operatorname{dim} u_{\left(\alpha_{i}\right)}=1$ for $\alpha_{i} \in M, \alpha_{i}$ is defined over $R$. Thus the restriction of $\alpha_{i} \in M$ to $T^{\prime}$ is the zero map. Let $\operatorname{dim} U_{\left(\alpha_{i}\right)}=1$. Then $U_{\left(\alpha_{i}\right)}$ is a root group for $T$ as well as a restricted root group of $S$. Thus for any root $\beta$ such that $\beta(S)=1, \alpha_{i}+n \beta$ is not a root and therefore $U_{\beta} \in C(S)$. The elements in $U_{\beta}$ with $\beta(S)=1$ commute with the elements in $U_{\left(\alpha_{i}\right)}$. Likewise, conjugation by an element $t \in T^{\prime}$ leaves $x_{\alpha_{i}}$ unchanged since $\alpha_{i}\left(T^{\prime}\right)=1$. Therefore, since $T^{\prime}$ and the $U_{\beta}$ generate $D(C(S))$, the elements of $D(C(S))_{R}$ commute with $x_{\alpha_{i}} \in U_{\left(\alpha_{i}\right)}$ where $\operatorname{dim} U_{\left(\alpha_{i}\right)}=1$. Thus an $R$-regular element of a connected component $A$ of $R_{u}$ is $G_{R}$-conjugate to an element of another connected component $B$ only if it is $S_{R}$-conjugate to some element of $B$.

If an $R$-regular unipotent element $x$ in a connected component $A$ of $R_{u}$ is $S_{R}$-conjugate to some element in another connected component $B$ then by Corollary $6, x$ is $G_{R}$-conjugate to all elements in $B$.

If $x$ is an element of $M_{i}$ and $s$ is an element of $S_{R}$ such that $\alpha_{i}(s) \in R^{-}$, the negative real numbers, then $s x s^{-1} \in N_{i}$, since

$$
x=x_{\alpha_{1}} x_{\alpha_{2}} \cdots x_{\alpha_{i}}(t) \cdots x_{\alpha_{n}} x_{\beta_{1}} \cdots x_{\beta_{m}}
$$

and

$$
s x s^{-1}=x_{\alpha_{1}}^{\prime} x_{\alpha_{2}}^{\prime} \cdots x_{\alpha_{i}}\left(\alpha_{i}(s) t\right) \cdots x_{\alpha_{n}}^{\prime} x_{\beta_{1}}^{\prime} \cdots x_{\beta_{m}}^{\prime} \in N_{i} \text {. }
$$

Likewise if $x$ is an element of $M_{i}$ and $s x s^{-1}$ is an element of $N_{i}$, then $\alpha_{i}(s)$ is negative. 
A connected component $A$ of $R_{u}$ equals $\bigcap_{i=1}^{p} M_{n_{i}} \cap \bigcap_{j=1}^{q} N_{n_{j}}$, since $A$ lies either in $M_{i}$ or $N_{i}$ for each $i . \rho_{i}(A)=\rho_{i}(B)$ if either both $A$ and $B$ lie in $M_{i}$ or both lie in $N_{i}$. Thus if $x$ is an element of $A$ and $s x s^{-1}$ is an element of $B$ for $s \in S_{R}$, then $\alpha_{i}(s)$ is positive if $\rho_{i}(A)=\rho_{i}(B)$ and negative if $\rho_{i}(A) \neq \rho_{i}(B)$.

THEOREM 1. Let $G$ be an adjoint semisimple algebraic group. The $R$ regular unipotent elements are $G_{R}$-conjugate.

PROof. Since two minimal parabolic subgroups are $G_{R}$-conjugate, it suffices to show that two $R$-regular unipotent elements in $U_{R}$ are $P_{R}$-conjugate. By Proposition 18, it is sufficient to show that given any partition of the set $M$ into two sets $I$ and $J$ there exists an element $s \in S_{R}$ such that $\alpha_{i}(s)$ is positive for $i \in I$ and $\alpha_{j}(s)$ is negative for $j \in J$.

Extend $S$ to maximal torus $T$ of $G$ defined over $R$. The characters of $S$ are restrictions of characters of $T$. The restricted roots of $S$ are restrictions of roots of $T$. The restricted simple roots of $S$ are restrictions of the simple roots of $T$. Since the group is adjoint, the simple roots of $T$ generate the character grcup of $T$. Therefore, the restricted simple roots of $S$ generate the character group of $S$.

Now $S_{R}=\operatorname{Hom}\left(X^{*}(S), R^{*}\right)$. Since the simple roots are free generators for $X^{*}(S)$, there exists $s_{i} \in S_{R}$, such that $\alpha_{i}\left(s_{i}\right)$ is negative and $\alpha_{j}\left(s_{i}\right)=1$ for $j \neq i$.

Thus given a partition $M=I \cup J$ where $I \cap J=\varnothing$, let $s=s_{r_{1}} s_{r_{2}} \cdots$ $s_{r_{p}}$ where $\alpha_{r_{i}} \in I$. Then

$$
\alpha_{i}(s)=\alpha_{i}\left(s_{r_{1}} s_{r_{2}} \cdots s_{r_{p}}\right)=\alpha_{i}\left(s_{r_{1}}\right) \alpha_{i}\left(s_{r_{2}}\right) \cdots \alpha_{i}\left(s_{r_{p}}\right)=\alpha_{i}\left(s_{i}\right)
$$

is negative if $i \in I$. But $\alpha_{j}(s)=1$ if $j \in J$.

Therefore, the $R$-regular unipotent elements form a single $G_{R}$-conjugacy class.

An equivalent theorem to Theorem 1 was proved in [8] by Rothschild from the Lie algebra point of view.

THEOREM 2. If $G$ is an $R$-split semisimple algebraic group, then the number of conjugacy classes of $R$-regular unipotent elements is given by $2^{q}$ where $q$ is the number of cyclic components of even order in a direct sum decomposition of $Z(G)$, the center of $G$.

Proof. As in Theorem 1, we are concerned with finding the number of orbits of $S_{R}$ acting on the set of connected components of $R_{u}$.

Let $\phi \in X^{*}(S)$. We define $\bar{\phi}: S_{R} \rightarrow Z_{2}$, by 


$$
\bar{\phi}(s)=\left\{\begin{array}{lll}
0 & \text { if } \phi(s) & \text { is positive, } \\
1 & \text { if } \phi(s) & \text { is negative. }
\end{array}\right.
$$

Define $\overline{X^{*}(S)}=\left\{\bar{\phi} \mid \phi \in X^{*}(S)\right\}$ and $\bar{\Delta}_{R}=\left\{\bar{\alpha} \mid \alpha \in \Delta_{R}\right\}$.

Since $S$ is a torus, $\overline{X^{*}(S)}$ can be viewed as $Z_{2}$-vector space of dimension $n=\operatorname{dim} S$ and $\overline{X^{*}(S)}=$ number of connected components of $R_{u}$. Likewise we can form $Z_{2}\left(\bar{\Delta}_{R}\right)$, the $Z_{2}$-vector space generated by $\bar{\Delta}_{R}$. Let $s \in S_{R}$. Then $\hat{s}(\bar{\phi})=\bar{\phi}(s)$ maps $Z_{2}\left(\bar{\Delta}_{R}\right)$ into $Z_{2}$.

$$
\hat{s}(\bar{\phi}+\bar{\psi})=(\bar{\phi}+\bar{\psi})(s)=\bar{\phi}(s)+\bar{\psi}(s)=\hat{s}(\bar{\phi})+\hat{s}(\bar{\psi}) .
$$

Thus each element of $S_{R}$ gives a linear functional on $Z_{2}\left(\bar{\Delta}_{R}\right)$ into $Z_{2}$ " Suppose $s_{1}$ and $s_{2}$ are elements such that $\hat{s}_{1}=\hat{s}_{2}$. Then $\bar{\alpha}_{i}\left(s_{1}\right)=\bar{\alpha}_{i}\left(s_{2}\right)$ for all restricted roots. Therefore $s_{1} A s_{1}^{-1}=s_{2} A s_{2}^{-1}$ where $A$ is a connected component of $R_{u}$.

The set $\hat{S}=\left\{\hat{s} \mid s \in S_{R}\right\}$ is a subspace of the dual of $Z_{2}\left(\bar{\Delta}_{R}\right)$, since $\hat{s}_{1}(\bar{\phi})+\hat{s}_{2}(\bar{\phi})=\bar{\phi}\left(s_{1}\right)+\bar{\phi}\left(s_{2}\right)=\bar{\phi}\left(s_{1} s_{2}\right)=\widehat{s_{1} s_{2}}(\bar{\phi})$. If $\hat{S}$ is not the space of linear functionals, there must exist $\bar{\phi}$ in $Z_{2}\left(\bar{\Delta}_{R}\right)$ such that $s(\bar{\phi})=\bar{\phi}(s)=0$ for all $s \in S_{R}$. But, then $\bar{\phi}=0$. Thus $\hat{S}$ is the dual space of $Z_{2}\left(\bar{\Delta}_{R}\right)$. Since the simple roots generate the full set of roots, a linear functional on $Z_{2}\left(\bar{\Delta}_{R}\right)$ is determined by its behavior on $\bar{\Pi}$.

Therefore, given a connected component $A$ of $R_{u}$ there is a one to one correspondence between the connected components $B$ obtainable from $A$ by conjugation by elements in $S_{R}$ and the dual space of $Z_{2}\left(\bar{\Delta}_{R}\right)$. But the order of $Z_{2}\left(\bar{\Delta}_{R}\right)$ equals the order of its dual.

Thus the number of conjugacy classes of $R$-regular unipotent elements is given by the order of $\overline{X^{*}(S) / Z_{2}}\left(\bar{\Delta}_{R}\right)$, which is equal to $2^{q}$ where $q$ is the $\operatorname{dim} \overline{X^{*}(S) / Z_{2}}\left(\bar{\Delta}_{R}\right)$.

Now $Z_{2}\left(\bar{\Delta}_{R}\right)=Z\left(\Delta_{R}\right) /\left(Z\left(\Delta_{R}\right) \cap 2 X^{*}(S)\right)$ and $\overline{X^{*}(S)}=X^{*}(S) / 2 X^{*}(S)$.

But by the second isomorphism theorem we get

$$
Z_{2}\left(\bar{\Delta}_{R}\right) \cong \frac{\left(Z\left(\Delta_{R}\right)+2 X^{*}(S)\right)}{2 X^{*}(S)}
$$

Therefore,

$$
\overline{X^{*}(S)} / Z_{2}\left(\bar{\Delta}_{R}\right) \cong \frac{X^{*}(S) / 2 X^{*}(S)}{\left(Z\left(\Delta_{R}\right)+2 X^{*}(S)\right) / 2 X^{*}(S)}=X^{*}(S) /\left(Z\left(\Delta_{R}\right)+2 X^{*}(S)\right) .
$$

By the fundamental theorem of finitely generated abelian groups, there exists a basis $\left\{\phi_{1}, \phi_{2}, \cdots, \phi_{n}\right\}$ of $X^{*}(S)$ such that $\left\{m_{1} \phi_{1}, m_{2} \phi_{2}, \cdots\right.$, $\left.m_{n} \phi_{n}\right\}$ is a basis for $Z\left(\Delta_{R}\right)$. If $m_{i}$ is odd, $\phi_{i} \in Z\left(\Delta_{R}\right)+2 X^{*}(S)$. If $m_{i}$ is 
even, $\phi_{i} \notin Z\left(\Delta_{R}\right)+2 X^{*}(S)$, but $2 \phi_{i} \notin Z\left(\Delta_{R}\right)+2 X^{*}(S)$. Hence $\left\{r_{1} \phi_{1}, r_{2} \phi_{2}\right.$, $\left.\cdots, r_{n} \phi_{n}\right\}$ is a basis for $Z\left(\Delta_{R}\right)+2 X^{*}(S)$ with $r_{i}=1$ if $m_{i}$ is odd, $r_{i}=2$ if $m_{i}$ is even.

The center of $G$ is isomorphic to $X^{*}(S) / Z\left(\Delta_{R}\right)$. The number of components of $\overline{X^{*}(S)} / Z_{2}\left(\bar{\Delta}_{R}\right)$ in the decomposition into a direct sum of cyclic groups is equal to the number of components $q$ of $Z(G)$ with even order. Thus the number of $G_{R}$-conjugacy classes of an $R$-regular unipotent element is $2^{q}$ which is the order of $\overline{X^{*}(S)} / Z_{2}\left(\bar{\Delta}_{R}\right)$.

For $R$-split simply connected almost simple groups of the given type, we have the following number of conjugacy classes of $R$-regular unipotent elements.

TABLE 1.

TYPE OF GROUP

$Z(G)$

$\begin{array}{ll}A_{n} & Z_{n+1} \\ B_{n} & Z_{2} \\ C_{n} & Z_{2} \\ D_{n} & Z_{4} \quad \text { if } n \text { is odd } \\ & Z_{2} \times Z_{2} \text { if } n \text { is even } \\ E_{6} & Z_{3} \\ E_{7} & Z_{2} \\ E_{8} & Z_{1} \\ F_{4} & Z_{1} \\ G_{2} & Z_{1}\end{array}$

NUMBER OF CONJUGACY CLASSES OF $R$-REGULAR UNIPOTENT ELEMENTS

1 if $n$ is even

2 if $n$ is odd

2

2

2 if $n$ is odd

4 if $n$ is even

1

2

1

1

1

THEOREM 3. Let $G$ be a nonsplit, semisimple group defined over $R$ with irreducible restricted root system. Then there is only one conjugacy class of $R$ regular unipotent elements except possibly in the three cases whose restricted Dynkin diagrams are the following:
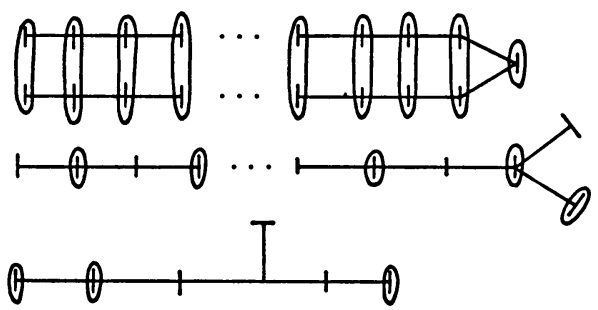

Proof. If $G$ is not absolutely almost simple, then $\operatorname{dim} U_{\left(\alpha_{i}\right)}>1$ for all $\alpha_{i} \in \Pi_{R}$ and $M=\varnothing$. Therefore, we can restrict ourselves to the absolutely almost simple case. 
The restrictions of roots and characters on a maximal torus $T$ defined over $R$ containing $S$ are restricted roots and characters respectively of $S$. Therefore, $X^{*}(S) / Z\left(\Delta_{R}\right)$ is a homomorphic image of $X^{*}(T) / Z(\Delta)$ where $\Delta$ is an absolute root system for $G$. Therefore, if $G$ is of type $E_{6}, E_{8}, F_{4}$ or $G_{2}, X^{*}(T) / Z(\Delta)$ is of odd order and hence $X^{*}(S) / Z\left(\Delta_{R}\right)$ is of odd order. Thus $\bar{\Pi}_{R}$ forms a basis for $\overline{X^{*}(S)}$. Therefore, for any subset $M \subseteq \Pi_{R}, \bar{M}$ can be extended to a basis of $\overline{X^{*}(S)}$.

For the remainder of the proof, we will show that in all other forms $M$ can be extended to a basis for $X^{*}(S)$, excluding the three possible exceptions.

Given a semisimple algebraic group $G$ defined over $R$, we can, by a construction of Borel and Tits in [3], construct a maximal split group $\widetilde{G}$ such that $S \subseteq G$ and $\alpha \in \Pi_{R}$ is a simple root of $\widetilde{G}$ if $2 \alpha \notin \Delta_{R}$ and if $2 \alpha \in \Delta_{R}$ then $2 \alpha$ is a simple root of $G$. Let $\Delta_{\tilde{G}}$ be the root system for $\widetilde{G}$. Let $V=$ $X^{*}(S) \otimes_{Z} R$. We can induce onto $V$ an inner product under the action of the Weyl group. To each element $\alpha \in \Delta_{\widetilde{G}}$, there exists a linear functional $\alpha^{*}$ on $V$ defined by $\alpha^{*}(v)=-2(\alpha, v) /(\alpha, \alpha)$. Note that $\alpha^{*}(\beta)$ is an integer for all $\alpha, \beta \in \Delta_{\widetilde{G}}$. The set of weights of the root system $\Delta_{\widetilde{G}}$ denoted $\Lambda_{\widetilde{G}}$ is the set $\left\{v \in V \mid \alpha^{*}(v) \in Z\right.$, for all $\left.\alpha \in \Delta_{\widetilde{G}}\right\}$. We can see that $\Lambda_{\widetilde{G}} \supseteq X^{*}(S) \supseteq \Delta_{\widetilde{G}}$. Therefore, it is sufficient to show that $M$ can be extended to a basis for $\Lambda_{\tilde{G}}$. Note that $M$ cannot contain $\alpha$ if $2 \alpha \in \Delta_{R}$, since $U_{(\alpha)}$ must be at least two dimensional. Therefore, $M \subseteq \Delta_{\widetilde{G}}$

The remainder of the proof will consist of checking the possible real forms to verify that $M$ can be extended to a basis for $\Lambda_{\widetilde{G}}$.

Let $\lambda_{\alpha}$ be the weight such that $\alpha^{*}\left(\lambda_{\alpha}\right)=1$ but $\beta^{*}\left(\lambda_{\alpha}\right)=0$ for $\alpha \neq \beta$ and $\alpha, \beta \in \Pi_{R}^{\prime}$, the set of simple roots of $\widetilde{G}$. Therefore, by examining the Dynkin diagram of $\widetilde{G}$, we can write each root of $\Pi_{R}^{\prime}$ in terms of the $\lambda_{\alpha}$ 's. It is clear that the $\lambda_{\alpha}$ 's freely generate $\Lambda_{\widetilde{G}}$. Therefore, we can determine from the expression of the roots of $M$ in terms of the $\lambda_{\alpha}$ 's, whether $M$ can be extended to a basis of $\Lambda_{\widetilde{G}}$. The restricted diagrams used in the following case by case study can be found in [12].

I. Let $G$ be of type $A_{n}$. The restricted Dynkin diagrams are:

(a)
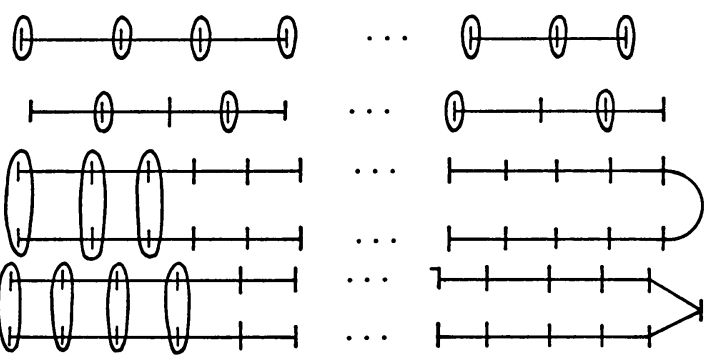
In case (a), $G$ is split. In case (b), $M=\varnothing$. In case (c), $M=\varnothing$. In case (d), $M=\varnothing$ except in the case which constitutes the first exception.

II. Let $G$ be of type $B_{n}$. The restricted Dynkin diagrams are:

(a)

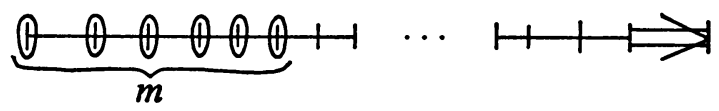

$\widetilde{G}$ is of type $B_{n}$ with diagram:

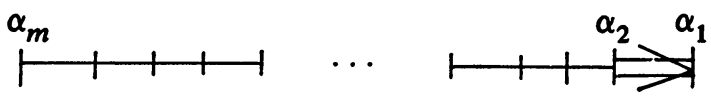

$M=\left\{\alpha_{2}, \alpha_{3}, \cdots, \alpha_{m}\right\}, \alpha_{m}=-2 \lambda_{\alpha_{m}}+\lambda_{\alpha_{m-1}}, \alpha_{i}=-2 \lambda_{\alpha_{i}}+\lambda_{\alpha_{i-1}}+\lambda_{\alpha_{i+1}}$ for $2 \leqslant i<m . M \cup \lambda_{\alpha_{m}}$ is a basis for $\Lambda_{\tilde{G}}$.

III. Let $G$ be of type $C_{n}$. The restricted Dynkin diagrams are:

(a)

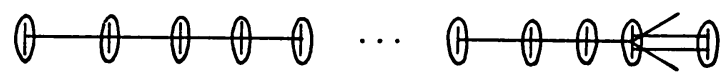

(b)

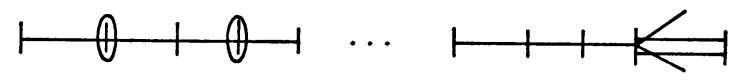

In case (a), $G$ is split. In case (b), $M=\varnothing$.

IV. Let $G$ be of type $D_{n}$. The restricted Dynkin diagrams are:

(a)

(b)

(c)

(d)
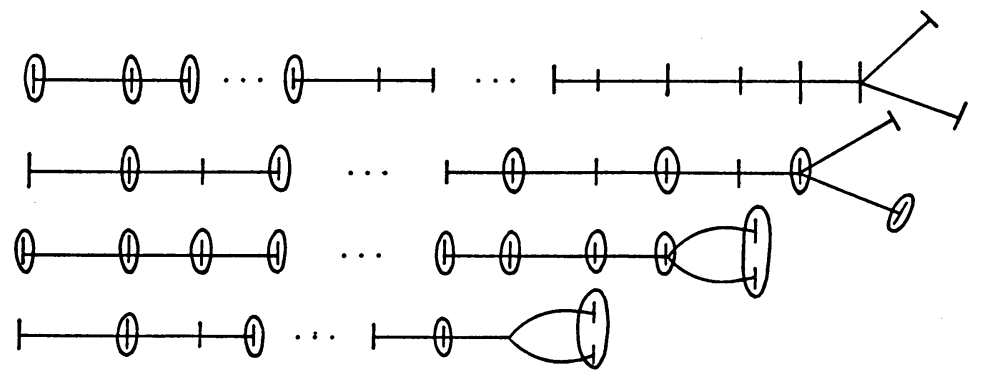

In case (a) either $G$ is split or $\widetilde{G}$ is of type $B_{n}$. In the latter case, the argument is the same as the above in type $B_{n}$. Case (b) is the second exception. In case (c), $\widetilde{G}$ is of type $B_{n}$ and the above argument again holds. In case (d), $M=\varnothing$.

V. Let $G$ be of type $E_{7}$. The restricted Dynkin diagrams are:
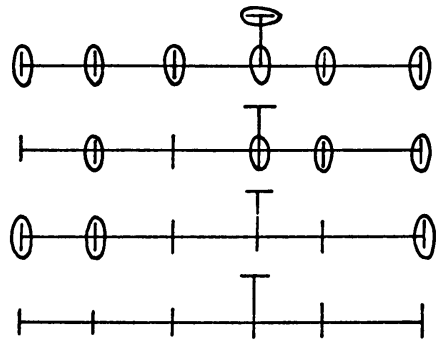
In case (a), $G$ is split. In case (b), $\widetilde{G}$ is of type $F_{4}$ and $\Lambda_{\widetilde{G}}=\Delta_{\widetilde{G}}$. Case (c) is the third exception. In case (d), $M=\varnothing$.

In each of the three exceptional cases $G$ is of type $C_{n}$ and $M$ consists of the unique long simple root, $\alpha_{m}$. In these cases, $\alpha_{m}=2 \lambda_{\alpha_{m-1}}-2 \lambda_{\alpha_{m}} \in$ $2 \Lambda_{\widetilde{G}}$. There are, therefore, at most two $G_{R}$-conjugacy classes of $R$-regular unipotent elements.

9. $Q_{p}$-conjugacy classes of $Q_{p}$-regular unipotent elements. For the following results, $k$ will be restricted to $Q_{p}$ the field of $p$-adic numbers.

Proposition 19. The $G_{Q_{p}}$-conjugacy class of a $Q_{p}$-regular unipotent element is open in $U_{Q_{p}}$, in the p-adic topology.

Proof. Let $u$ be a $Q_{p}$-regular unipotent element in $U_{Q_{p}}$. Then $u=$ $\exp (N)$. From the argument of Proposition 16, we see that ad $N\left(\mathfrak{p}_{Q_{p}}\right)=\mathfrak{p}_{Q_{p}}$. Thus if $\phi: P_{Q_{p}} \rightarrow U_{Q_{p}}$ with $\phi(p)=p u p^{-1}$ then the differential of $\phi$ is onto $\mathfrak{n}_{Q_{p}}$. But by $L G 3.15$ of [10], $\phi$ maps $P_{Q_{p}}$ onto an open set in $U_{Q_{p}}$.

Proposition 20. Let $x$ and $y$ be two $Q_{p}$-regular unipotent elements in $U_{Q_{p}}$ such that $x=x_{\alpha_{1}} x_{\alpha_{2}} \cdots x_{\alpha_{n}} x_{\beta_{1}} \cdots x_{\beta_{m}}$ and $y=y_{\alpha_{1}} y_{\alpha_{2}} \cdots y_{\alpha_{n}} y_{\beta_{1}}$ $\cdots y_{\beta_{m}}$ where $x_{\alpha_{i}} \neq e \neq y_{\alpha_{i}}$ for all $\alpha_{i} \in \Pi_{Q_{p_{1}}}$ and $\beta_{i}$ ranges over higher roots. $x$ is $G_{Q_{p}}$-conjugate to $y$ if and only if $x^{\prime}=x_{\alpha_{1}} x_{\alpha_{2}} \cdots x_{\alpha_{n}}$ is $C(S)_{Q_{p}}$ conjugate to $y^{\prime}=y_{\alpha_{1}} y_{\alpha_{2}} \cdots y_{\alpha_{n}}$ where $C(S)$ is the centralizer of the maximal $Q_{p}$-split torus $S$.

Proof. By the proof of Proposition 10, $x$ is $U_{Q_{p}}$-conjugate to $x^{\prime}$ and $y$ is $U_{Q_{p}}$-conjugate to $y^{\prime}$. Thus if $x^{\prime}$ is $C(S)_{Q_{p}}$-conjugate to $y^{\prime}$, then $x$ is $G_{Q_{p}}$-conjugate to $y$.

If $x$ is $G_{Q_{p}}$-conjugate to $y$ then by the argument of Proposition 17, $x$ is $P_{Q_{p}}$-conjugate to $y$ where $P$ is the unique minimal parabolic subgroup containing $x$ and $y$. Thus $x^{\prime}$ is $P_{Q_{p}}$-conjugate to $y^{\prime}$. But $P=C(S) U$ and the conjugation of $x^{\prime}$ with an element in $U_{Q_{p}}$ cannot leave an element whose only components are in simple root spaces unless the element in $U_{Q_{p}}$ commutes with $x^{\prime}$. Since conjugation by $C(S)$ normalizes the restricted root groups, the only elements of $P_{Q_{p}}$ which could conjugate $x^{\prime}$ into $y^{\prime}$ are of the form $c u$ where $c \in C(S)_{Q_{p}}, u \in U_{Q_{p}}$ and $u$ commutes with $x^{\prime}$. But then $c x c^{-1}=y^{\prime}$ and $x^{\prime}$ and $y^{\prime}$ are $C(S)_{Q_{p}}$-conjugate.

THEOREM 4. There are only a finite number of $G_{Q_{p}}$-conjugacy classes of $Q_{p}$-regular unipotent elements.

Proof. From Proposition 20, it suffices to show that there are only a finite number of orbits of $C(S)_{Q_{p}}$ acting on $R=\left\{u \mid u=x_{\alpha_{1}} x_{\alpha_{2}} \cdots x_{\alpha_{n}}\right.$, 
$\alpha_{i} \in \Pi_{Q_{p}}$ and $x_{\alpha_{i}} \neq e$ for each $\alpha_{i} \in \Pi_{Q_{p}}$ \}. Topologically, $R=U_{\alpha_{1} Q_{p}}^{\prime} \times$ $U_{\alpha_{2} Q_{p}}^{\prime} \times \cdots \times U_{\alpha_{n} Q_{p}}^{\prime}$ where $U_{\alpha_{i} Q_{p}}^{\prime}=U_{\alpha_{i} Q_{p}}-\{e\}$. Let $M_{\alpha_{i} Q_{p}}$ be the projective space produced from $U_{\alpha_{i} Q_{p}}^{\prime}$ by identifying all points lying on a line through the origin. $C(S)_{Q_{p}}$ operates on $M_{\alpha_{i} Q_{p}}$ and hence on $M_{Q_{p}}=$ $\Pi_{i=1}^{n} M_{\alpha_{i} Q_{p}}$. Since $\alpha_{i}$ is an integral combination of basis elements in $X^{*}(S)$, $Q_{p}^{*} / \alpha_{i}\left(S_{Q_{p}}\right)$ is finite. Therefore, if $\phi: \prod_{i=1}^{n} U_{\alpha_{i} Q_{p}}^{\prime} \rightarrow M_{Q_{p}}$ is the projection map, then an orbit in $M_{Q_{p}}$ has only a finite number of orbits in its preimage in $R$. Since $M_{\alpha_{i} Q_{p}}$ is compact, $M$ is compact. The orbits of $C(S)_{Q_{p}}$ are open in $R$, hence they are open in $M$. Therefore, there are only a finite number. Thus there are only a finite number of $G_{Q_{p}}$-conjugacy classes of $Q_{p}$-regular unipotent elements.

I wish to thank Professor Mostow, Professor Tamagawa and Professor Seligman for their kind help.

\section{REFERENCES}

1. P. Andre, $k$-regular elements in semisimple algebraic groups, Ph. D. Dissertation, Yale University, New Haven, Conn., 1970.

2. A. Borel, Linear algebraic groups, Benjamin, New York, 1969. MR 40 \#273.

3. A. Borel and J. Tits, Groupes réductifs, Inst. Hautes Études Sci. Publ. Math. No. 27 (1965), 55-150. MR 34 \#7527.

4. N. Jacobson, Lie algebras, Interscience Tracts in Pure and Appl. Math., no. 10, Interscience, New York, 1962. MR 26 \#1345.

5. B. Kostant, The principal three-dimensional subgroup and the Betti numbers of a complex simple Lie group, Amer. J. Math. 81 (1959), 973-1032. MR 22 \#5693.

6. B. Kostant and S. Rallis, Orbits and representations associated with symmetric spaces, Amer. J. Math. 93 (1971), 753-809. MR 47 \#399.

7. D. Mumford, Introduction to algebraic geometry, Harvard University, Cambridge, Mass. (preliminary edition).

8. L. P. Rothschild, Orbits in a real reductive Lie algebra, Trans. Amer. Math. Soc. 168 (1972), 403-421.

9. Séminaire C. Chevalley 1956-1958: Classification des groupes de Lie algébriques, Two volumes, Secrétariat mathématique, Paris, 1958. MR 21 \#5696.

10. J.-P. Serre, Lie algebras and Lie groups, Benjamin, New York, 1965. MR 36 \#1582.

11. R. Steinberg, Regular elements of semisimple alge braic groups, Inst. Hautes Etudes Sci. Publ. Math. No. 25 (1965), 49-80. MR 31 \#4788.

12. J. Tits, Classification of algebraic semisimple groups, Proc. Sympos. Pure Math., vol. 9, Amer. Math. Soc., Providence, R. I., 1966, pp. 33-62. MR 37 \#309.

13. - Lectures on algebraic groups, Yale University, New Haven, Conn., 1967.

DEPARTMENT OF MATHEMATICS, WILLIAMS COLLEGE, WILLIAMSTOWN, MASSACHUSETTS 01267

Current address: Department of Mathematics, United States Naval Academy, Annapolis, Maryland 21402 